\title{
A general model to simulate how an invading organism's dispersal characteristics influence its spread, and the implications for surveillance strategies
}

\author{
$\underline{\text { M. D. Triska }}^{\mathrm{a}, \mathrm{b}}$ and M. Renton ${ }^{\mathrm{a}, \mathrm{b}}$ \\ a School of Plant Biology, The University of Western Australia \\ ${ }^{b}$ Plant Biosecurity Cooperative Research Centre, Australian Capital Territory \\ Email:maggie.triska@uwa.edu.au
}

\begin{abstract}
Invading organisms do not all exhibit the same dispersal characteristics. Some may spread at a constant rate across the landscape, in a process similar to diffusion, typically modelled with 'thin-tailed' or non-leptokurtic dispersal kernels. For others, dispersal may include occasional long-distance dispersal events that allow them to quickly expand into new areas at a rate that increases with time. This kind of dispersal is typically modeled with 'fat-tailed' or leptokurtic dispersal kernels. These varying dispersal characteristics are likely to affect the dynamics and spatial patterns of spread of a new invader, and are thus likely to have implications for the effectiveness of surveillance strategies.
\end{abstract}

Spread models allow us to predict the spread of invasive organisms to help evaluate the efficacy of different surveillance strategies. Many spread models assume non-leptokurtic, constant-rate diffusive spread, possibly because including long-distance dispersal events through leptokurtic dispersal kernels adds to the computational complexity of the spread model. Modelling only non-leptokurtic, constant-rate dispersal, risks overlooking important aspects of the dynamic nature of how real organisms spread. This may in turn affect the results when the model is used to assess surveillance strategies. In particular, we predicted that models that do not incorporate leptokurtic dispersal might underestimate the spread of an invasion and thus overestimate the efficacy of standard surveillance strategies. Therefore, the aim of this study was to design a general model of the spread of a new biological invasion that accounts for varying dispersal characteristics, including both leptokurtic and non-leptokurtic dispersal. Then use this model to assess how the dispersal characteristics influence the dynamic and spatial patterns of spread, and provide insights into the potential implications of leptokurtic dispersal for surveillance strategies.

We simulated the spread of a new biological invasion using two types of dispersal kernels, a non-leptokurtic kernel (exponential) and a leptokurtic kernel (Weibull) with varying degrees of leptokurtosis. We then assessed if the spread of the invasion and its potential to move beyond the simulated area differed between the dispersal types and distances over the same time period. We showed that over the same time period, leptokurtic dispersal models predicted spread over greater distances and area than the non-leptokurtic dispersal models. This suggests that surveillance options will need to be different in regards to survey density and frequency in order to detect the invasions at a similar extent of spread. We also determined that species with high degrees of leptokurtic dispersal may be difficult to contain with buffers around an invasion site, as they consistently moved beyond the simulated area. Non-leptokurtic and leptokurtic models with low degrees of long-distance dispersal did not often, if ever, go beyond the simulated area, suggesting that organisms with these dispersal characteristics can be contained with a buffer. The dispersal type and distance of the invading organism impacted its spread, and will likely impact the efficacy of different surveillance strategies. Thus, when using spread models to evaluate and improve surveillance strategies, it is important to simulate the spread of invasions with models that account for species-specific dispersal characteristics.

Keywords: $\quad$ Biosecurity, diffusion, dispersal kernels, invasion biology, leptokurtic 
Triska and Renton, A general model to simulate how an invading organism's dispersal characteristics influence its spread, and the implications for surveillance strategies

\section{INTRODUCTION}

The number of biological invasions is increasing and these new invasions are often associated with negative impacts (Slimberloff et al., 2013). These negative impacts include economic loss due to trade restrictions, reductions in biodiversity and high management costs. To reduce these impacts, it is essential to prepare for new invasions, even though the initial location of an invasion is difficult, if not impossible, to predict. Therefore, to prepare for new invasions it is important to have a spread model that can simulate potential invasions prior to their occurrence. A general spread model simulates dispersal, and subsequent spread, that is specific to an invasion and accounts for an organism's biological characteristics and the specific landscape. A flexible and realistic spread model can also help improve surveillance strategies and thus the ability of incursion managers to detect and potentially eradicate the invasion before it has spread too far.

Biological and particularly dispersal characteristics, are important in determining how a new biological invasion will spread. Theoretical models that define species' spread often use non-leptokurtic diffusion equations to simulate dispersal (Skellam, 1951; Suarez et al., 2001). This is possibly due to the added complexity of incorporating leptokurtic dispersal into such models. Diffuse spread alone may represent the spread of most organisms well, at least initially, but new invasions often include leptokurtic or fat-tailed dispersal (Kot et al., 1996; Lindström et al., 2011). Leptokurtic dispersal results in the rate of spread increasing with time due to occasional random long-distance dispersal events (Hastings et al., 2005; Kot et al., 1996; Nathan et al., 2012). In reality, these long-distance dispersal events may be part of the natural movement of the organism, but are often assisted by external human vectors or extreme environmental events.

Spread models that only assess diffuse spread may underestimate the actual spread of the invasion and neglect the dyanamic nature of how organisms spread (Hengeveld, 1989; Higgins and Richardson, 1999; Kot et al., 1996; Suarez et al., 2001). The consequences of underestimating the spread of new invasions, and using the generated 'reduced' spread predictions in additional models is unknown. Therefore, simulation models can be used to help evaluate the efficacy of different surveillance strategies by allowing for the generalization and isolation of factors that may not be feasible in the real landscape. They enable the simulation of multiple dispersal characteristics and their spread, which are not possible prior to an invasion occurring in a landscape. Subsequently, a general spread model, which accounts for dispersal characteristics can be generated to assess the impact of an organism's dispersal characteristics on the time to detection and total incursion spread across multiple surveillance strategies. This is particularly important for invasive species that do not yet occur in a region (Peck, 2004).

Our study aimed to build a general spread model to simulate the spread of a new biological invasion via multiple dispersal types and distances to assess the influence of dipsersal on the dynamic and spatial patterns of a new biological invasion. We simulated the spread of four new biological invasions using two types of dispersal kernels, a non-leptokurtic kernel (exponential) and a leptokurtic kernel (Weibull) with varying degrees of leptokurtosis. We measured the potential influence of dispersal characteristics, including the type (nonleptokurtic exponential or leptokurtic Weibull) and median dispersal distance (for Weibull distributions) on the spread of the invasion over time and the ability of the invasion to move beyond the simulated area. We predicted that non-leptokurtic distributions will have reduced spread (i.e. infect a smaller area and go beyond the study area fewer times) compared to all leptokurtic distributions over the same time period. Additionally, if nonleptokurtic and leptokurtic models have different spread patterns and rates, they will likely require different surveillance strategies such as survey frequency, arrangement or density.

\section{GENERAL SPREAD MODEL}

The general spread model we developed simulates the spread of a new biological invasion by a pest that undergoes passive or non-directed dispersal, such as a soil borne organism, in a farm landscape. The model, implemented in the R 3.1.2 software environment, represents the invasive pest reproducing and dispersing over a user-specified number of time steps. The landscape is represented as a grid of square cells or pixels that are designated as suitable or non-suitable for pest occupancy. Reproduction within a pixel is defined by a production function: 
Triska and Renton, A general model to simulate how an invading organism's dispersal characteristics influence its spread, and the implications for surveillance strategies

$$
\begin{array}{ll}
\operatorname{prod}(t)=0, & \text { if } t \leq t_{0} \\
\operatorname{prod}(t)=r_{0} *\left(t-t_{0}\right)+\left(\frac{0.5 * p_{\max }-r_{0} *\left(t_{50}-t_{0}\right)}{\left(t_{50}-t_{0}\right)^{2}}\right) *\left(t-t_{0}\right)^{2}, & \text { if } t>t_{0} \text { and } t \leq t_{50} \\
\operatorname{prod}(t)=0.5 * \operatorname{p}_{\text {max }} *\left(1+\left(\frac{r_{0}+2 *\left(\frac{0.5 * p_{\max }-r_{0} *\left(t_{50}-t_{0}\right)}{\left(t_{50}-t_{0}\right)^{2}} *\left(t-t_{0}\right)\right) *\left(t-t_{50}\left(t-t_{50}\right)\right)}{r_{0}+2 *\left(\frac{0.5 * p_{\max }-r_{0} *\left(t_{50}-t_{0}\right)}{\left(t_{50}-t_{0}\right)^{2}} *\left(t-t_{0}\right)\right) *\left(t-t_{50}\left(t-t_{50}\right)\right)}\right)\right), & \text { if } t>t_{50}
\end{array}
$$

where $\operatorname{prod}(t)$ is the number of new propagules produced, $t$ is the time since that cell was first infested, and the parameters represent the maximum number of offspring produced each time step $\left(p_{\max }\right)$, the time at which reproduction begins $\left(t_{0}\right)$, the initial rate at which production increases $\left(r_{0}\right)$ and the time at which $50 \%$ of the maximum production rate is reached $\left(t_{50}\right)$ (Figure 1).

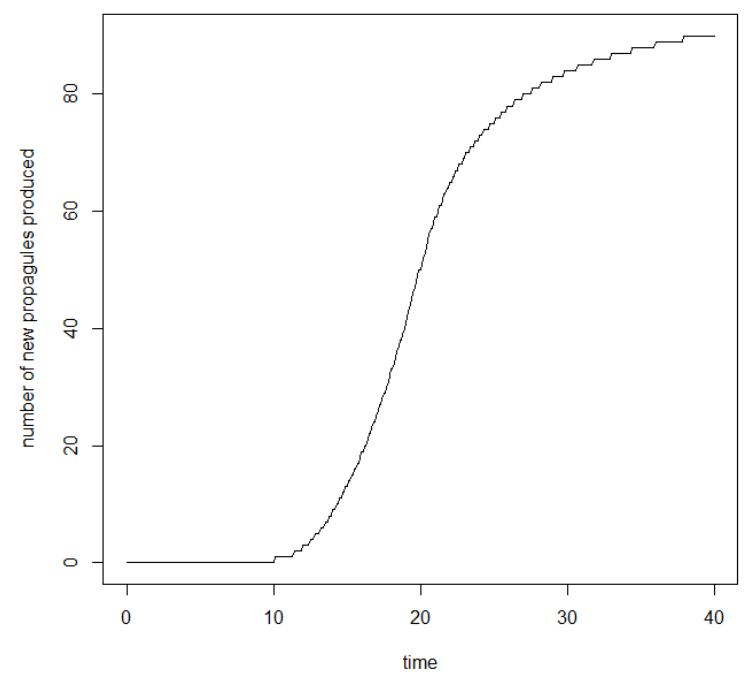

Figure 1. Example of the production function output with $\mathrm{p}_{\max }=100, \mathrm{t}_{0}=10, \mathrm{r}_{0}=0.05$ and $\mathrm{t}_{50}=20$.

The production function values $\left(n_{\text {prod }}\right)$ are used to determine total number of offspring that can establish $\left(n_{\text {estab }}\right)$. They are created by randomly selecting the same number of values contained in $n_{\text {prod }}$ from a bionmial distribution with size $n_{\text {prod }}$ and proportion of offspring that can establish $\left(p_{\text {estab }}\right)$. The probability of a new infestation is dependent on the relative population size of the source (via the production function) and the distance from the source (via a dispersal kernel). Any dispersal kernel can be used.

\subsection{Simulations}

For this study we simulated a new invasion on a 614 x $614 \mathrm{~m}$ (37.7 ha) farm, with 1 x $1 \mathrm{~m}$ square pixels. The simulated area was divided into 25 fields, each $100 \times 100 \mathrm{~m}$ (1 ha). The simulated fields have narrow divisions ( 2 $\mathrm{m}$ ) between them that represent non-host plants, unplanted fencelines, or roads, and there is a $50 \mathrm{~m}$ buffer around the fields (Figure 2a). We assumed that the pest cannot establish when host plants are not present (i.e. in divisions between fields and the buffer).

We conducted a series of simulations to examine how dispersal characteristics of an invading organism affect its spread. We compared one exponential and three Weibull dispersal kernels (Exp, Wei05, Wei10, Wei20) (Table 1). For each kernel we conducted 100 replicate simulation runs, with each run simulating spread over 200 time steps (nominally representing months) (Table 2). For each simulation run, a pixel was randomly selected from one of the 25 fields to represent the initial location of the invasion. Spread from this initial site was simulated, with the organism producing propagules based on the production function. Propagules dispersed according to the dispersal kernel and all simulations used the same production function (Figure 1).

For each dispersal kernel, random deviates were generated from exponential and Weibull distributions to generate the dispersal function. The dispersal kernel parameters include the median dispersal distance (i.e. the distance travelled by $50 \%$ of the pests: $d_{50}$ ) and a maximum dispersal distance (i.e. the maximum distance travelled by $1 \%$ of the pests: $d_{99}$ ). The $d_{50}$ and $d_{99}$ values determine the tail of the model, and if the $k$ value is $<1$ the distribution is fat-tailed. Expert knowledge and information on dispersal is required to determine the mean and tails of the 
Triska and Renton, A general model to simulate how an invading organism's dispersal characteristics influence its spread, and the implications for surveillance strategies

dispersal kernels. In the exponential model the rate parameter was held constant $\left(d_{50}=0.50\right)$. In the Weibull models the scale $(k)$ and shape $(\lambda)$ parameters varied and $k$ was defined by the $d_{50}$ value, and $\lambda$ by the $d_{99}$ and $d_{50}$
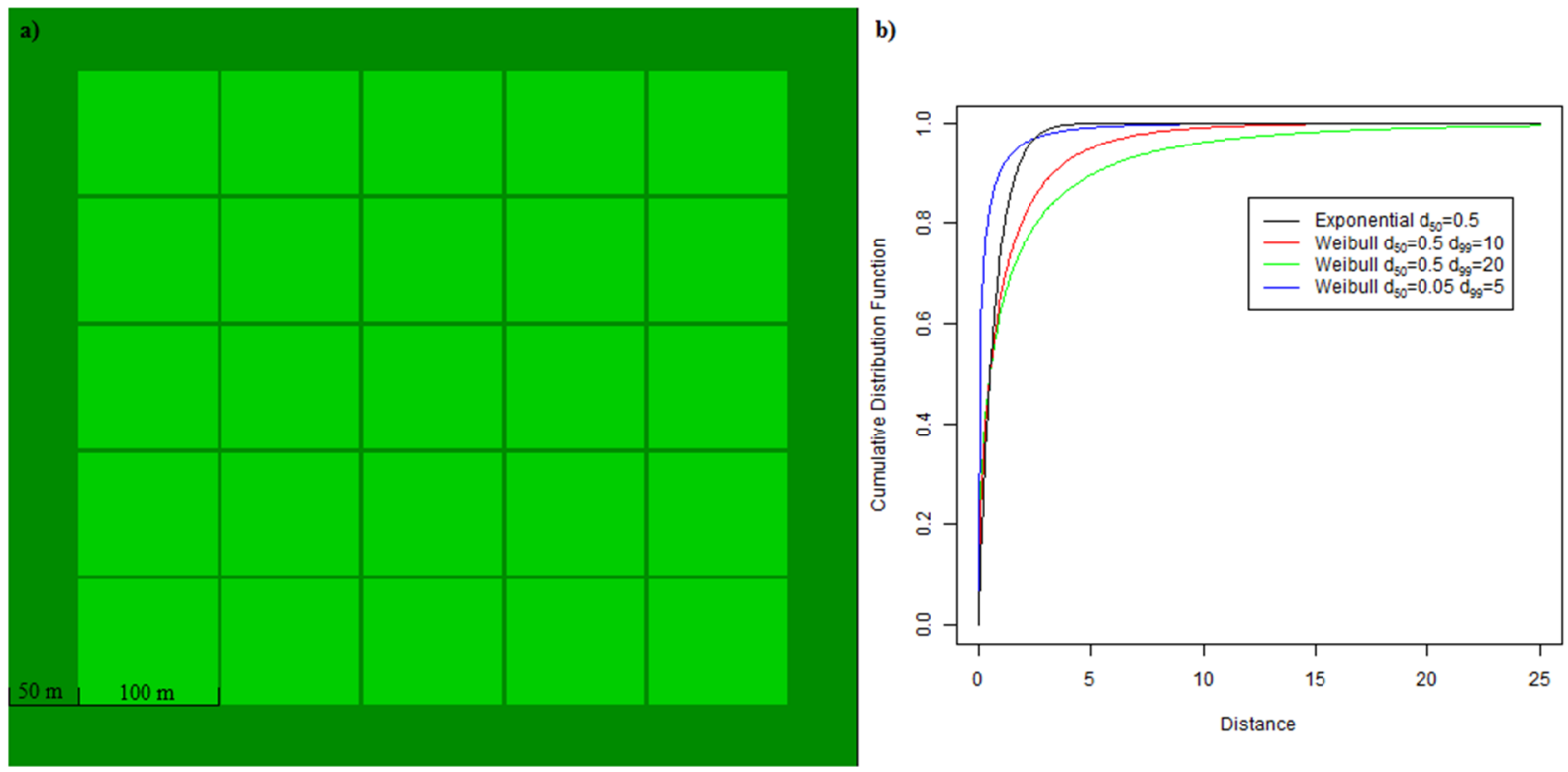

Figure 2. The landscape over which the invading organism's spread was simulated; (a) it simulates an area or farm of $614 \times 614 \mathrm{~m}$, individual fields are $100 \times 100 \mathrm{~m}$, and a $50 \mathrm{~m}$ buffer is located around the farm and (b) displays the four different dispersal kernels used to simulate the spread of a new invasion (exponential and Weibull) and their dispersal characteristics (Exponential [Exp]; $\mathrm{d}_{50}=0.5$, Weibull [Wei5]; $\mathrm{d}_{99}=5$ and $\mathrm{d}_{50}=0.05$, Weibull [Wei10] $10 ; \mathrm{d}_{99}=10$ and $\mathrm{d}_{50}=0.5$, and Weibull [Wei20]; $\mathrm{d}_{99}=20$ and $\mathrm{d}_{50}=0.5$ ).

values (Figure $2 \mathrm{~b}$ and Table 1$)$. Two of the Weibull models had the same median $\left(d_{50}\right)$ as the exponential model, but possessed an added potential for long-distance dispersal events of 10 and $20 \mathrm{~m}$. In the remaining Weibull model (Wei05) the median and long-distance dispersal values were decreased to mimic the spread rate observed in the exponential model (Figure 2). All the models have a small natural dispersal kernel and only spread long distances assisted by human or environmental vectors in the three leptokurtic models. The fat-tail of the leptokurtic distributions resulted in individuals dispersing outside of the simulated area (i.e. beyond the fields and $50 \mathrm{~m}$ buffer around the them) and the number of these dispersal events was recorded during each simulation.

Table 1. The dispersal kernels used in this study (Exp: $d_{50}=0.50$; Wei5: $d_{99}=5.00$ and $d_{50}=0.05$; Wei10: $d_{99}=10.00$ and $d_{50}=0.50$; and Wei20: $d_{99}=20.00$ and $d_{50}=0.50$ ).

\begin{tabular}{lcc} 
& Dispersal Kernel & Parameters \\
\hline Exponential & $f(\mathrm{x})=r e^{-r \mathrm{x}}$ & $r=\log (2) / d_{50}$ \\
& & $k=\log \left(\left(\frac{\log (100)}{\log (2)}\right) / \log \left(d_{99} / d_{50}\right)\right)$ \\
Weibull & $f(\mathrm{x})=k \lambda \mathrm{x}^{k-1} e\left(-\lambda \mathrm{x}^{k}\right)$ & $\lambda=d_{50} \times \log (2)^{(-1 / k)}$ \\
\hline
\end{tabular}

Table 2. The input variables required for the general spread model, with the model outputs in bold.

\begin{tabular}{lll} 
Symbol & Explanation & Units* \\
\hline$p_{\max }$ & Maximum number offspring/generation & 10000 \\
$r_{0}$ & Rate of spread & $1.0 \mathrm{~m} /$ year \\
$t_{0}$ & Start of reproduction & 5 months \\
$t_{50}$ & Time at which $50 \%$ of the offspring max is reached & 15 months \\
$p_{\text {estab }}$ & Proportion of offspring that establish & 0.01 \\
$d_{50}$ & Distance traversed by $50 \%$ of the pests & 0.05 or $0.5 \mathrm{~m}$ \\
$d_{99}$ & Distance traversed by $99 \%$ of the pests & 5,10 or $20 \mathrm{~m}$ \\
out & Number that go beyond the simulated area & $\mathbf{0}$-inf \\
$\boldsymbol{t}$ & Time to detection & $\mathbf{0 - 2 0 0}$ months \\
\hline
\end{tabular}

*The specific units of each measurement can be defined by the user 
Triska and Renton, A general model to simulate how an invading organism's dispersal characteristics influence its spread, and the implications for surveillance strategies

\section{INITIAL RESULTS}

The different dispersal kernels resulted in different patterns of spread over time (Figures 3 and 4 ). The spread of organisms with non-leptokurtic, exponential dispersal moved beyond one field on average after 88 time steps (mean of 100 replicate runs), whereas the spread of organisms with leptokurtic, Weibull dispersal moved beyond one field after an average of 27 time steps (mean of 100 replicate runs). There were also differences in dispersal distances within dispersal types. For example at time step 50, in the exponential model (mean $\pm \mathrm{SE}$ ) $1.45 \pm 0.06$ fields were invaded, in the Wei05 $3.83 \pm 0.12$ fields were invaded, in the Wei10 $4.24 \pm 0.15$ fields were invaded and in the Wei20 $12.26 \pm 0.39$ were invaded. At time step 150, in the exponential model $2.98 \pm$ 0.09 fields were invaded, in the Wei05 $14.43 \pm 0.36$ fields were invaded, in the Wei10 $14.12 \pm 0.41$ fields were invaded and in the Wei20 25.00 \pm 0.00 fields were invaded. By approximately 100 time stepss, the models with the highest degree of leptokurtic dispersal (Wei20) were infested in all fields $(n=25)$.

Invasions were counted as moving beyond the simulated area when organisms dispersed beyond the outer $50 \mathrm{~m}$ buffer. Invasions commonly dispersed beyond the simulated area in the Wei20 models, but this long-distance movement was limited or non-existent in all other models (Figure 4). The Exp models had no invasions that dispersed beyond the simulated area; however, in all Weibull models some invasions dispersed beyond the simulated area. On average the first invasions to disperse beyond the simulated area in the Wei05 and Wei10 kernels occurred at similar time steps (30 and 31, respectively), whereas the Wei20 kernels dispersed beyond the simulated area after time step 16. After 150 time steps, the number of invasions (mean $\pm \mathrm{SE}$ ) dispersing beyond the simulated area was $12.89 \pm 0.85$ for the Wei05, 3.91 \pm 0.28 for the Wei10 and 11,866.12 \pm 215.14 for the Wei20. The long-distance dispersal events in the Weibull dispersal kernels resulted in changes in the pattern of spread to include satellite populations, which did not occur in the exponential dispersal kernel (Figures 3 and 4).
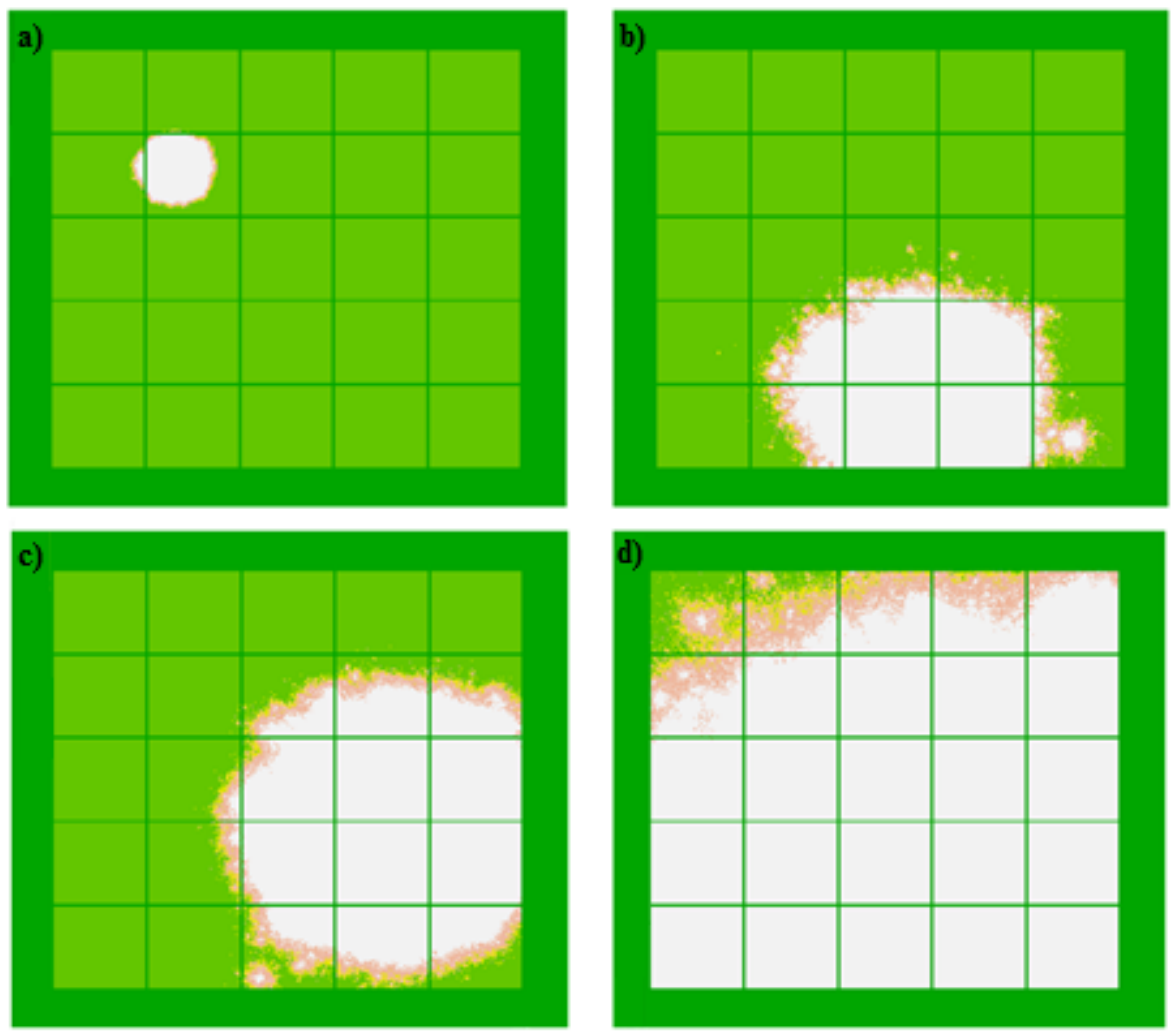

Time step $=150$

Figure 3. Initial model output (a) shows spread via exponential dispersal kernel while spread via Weibull dispersal kernels [(b) Wei05, (c) Wei10 and (d) Wei20]. All model outputs represent spread at time step 150, and it can be seen that the invasion has spread further over the simulated area (i.e., infested more fields and more total area) with the Weibull dispersal kernels (b, c, d) than with the exponential dispersal kernel (a). 
Triska and Renton, A general model to simulate how an invading organism's dispersal characteristics influence its spread, and the implications for surveillance strategies
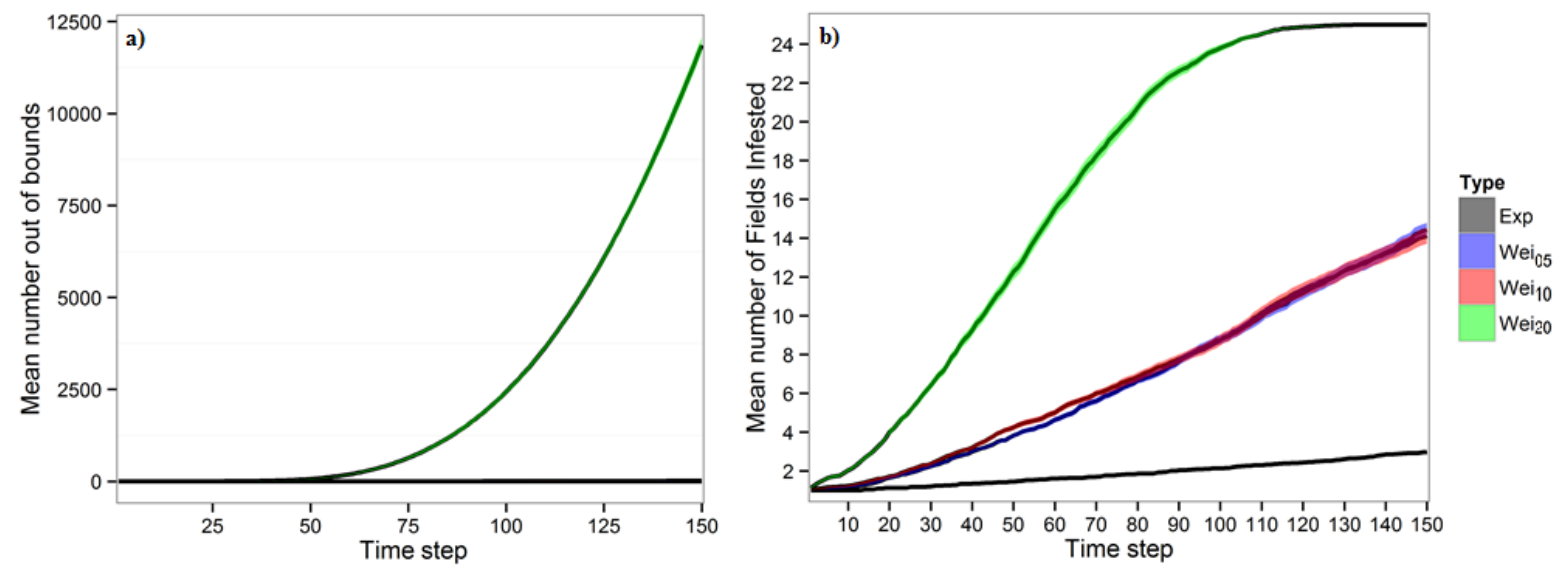

Figure 4. The number of times the invading organism dispersed (a) beyond the $50 \mathrm{~m}$ buffer around the farm and (b) the total number of fields infested at each time step within a simulated invasion. The solid lines indicate the means of 100 replicate runs and the shaded ribbon indicates the standard error of the mean.

\section{CONCLUSIONS AND RECOMMENDATIONS}

We designed a general model that can be used to simulate how different dispersal characteristics affect the dynamic spatial spread patterns of new biological invasions, and applied the model to a case study of an invasion on a farm. The results support our initial prediction that dispersal characteristics such as dispersal type and distance strongly influence the spread of an invasion. Specifically, we determined that the spread of an organism is reduced in non-leptokurtic models compared to leptokurtic models, based both on the number of fields infested and the number of times the invasion went beyond the outer $50 \mathrm{~m}$ buffer. This suggests that dispersal type and distance should be taken into account when modelling the spread of an invasion and defining optimal surveillance strategies and other management or control procedures.

Prior to establishment, invading organisms' spread can be represented by an exponential dispersal kernel (Mehta et al., 2007). However, invasions are not always detected early on, and the organism likely to become established prior to detection. Consequently, even if initially species exhibit exponential spread (Mehta et al., 2007), incorporating leptokurtic dispersal into spread models provides an improved model of spread for invasions that have established prior to detection. The addition of leptokurtic dispersal improves the prediction of spread at the time of detection and will likely improve surveillance, eradication or control strategies.

After an invasion has been detected, it is important to limit or contain the invasion from spreading to neighbouring areas. In our models, a $50 \mathrm{~m}$ buffer around the farm was large enough to restrict the dispersal of an invading organism with non-leptokurtic dispersal and reduce the potential for long-distance spread of organisms with low degrees of leptokurtic dispersal (Wei10 and Wei05). Contrarily, an organism with a high degree of leptokurtic dispersal (Wei20) was able to spread beyond the buffer easily, and the number that dispersed beyond the buffer increased exponentially over time. Therefore, species that are dispersed over short distances (i.e. by soil adhering to farm workers shoes or equipment) may be contained within a field or farm if adequate buffers coupled with sanitation, cleaning and safety measures are followed. Containment of an invasion may not be possible if organisms are transported further (i.e. via trucks or during storms with strong winds, or appropriate quarantine hygiene methods are not effectively employed) then different surveillance and management options would be required.

Optimising surveillance and management strategies is key to any biosecurity program. Surveillance strategies such as survey density and frequency help to assist in the detection of invasions soon after their arrival. We suggest that organisms with non-leptokurtic and leptokurtic dispersal, are likely to require different surveillance strategies. For example, organisms with non-leptokurtic dispersal move slowly from the initial infestation and their optimal surveillance strategy may rely on increased survey density to detect the slowly moving, concentrated incursion. Contrarily, invasions with leptokurtic dispersal spread quickly and surveillance strategies may be improved by increasing the sampling frequency and survey area to detect the invasion prior to its expansion into other areas. However, the inferences based on our results and dispersal type requires further assessment via simulation models that assess different surveillance strategies. 
Triska and Renton, A general model to simulate how an invading organism's dispersal characteristics influence its spread, and the implications for surveillance strategies

In this study, our initial aim was to make the Wei05 model mimic the overall spread rate of the Exp model; however, despite the much lower $d_{50}$ value for the Wei05 model than for the Exp model, the rate of spread in the Wei05 model was much higher than for the Exp model. Prior to moving on to the assessment of surveillance strategies, we plan to develop an additional exponential dispersal model with similar overall spread rates to the Wei05 and Wei10 models so that we can more fairly compare leptokurtic vs non-leptokurtic spread.

Following the development of another exponential model more directly comparable to the Weibull models interms of spread rate, we will use the models to evaluate different surveillance strategies including various surveillance arrangements (grid vs random), densities (the number of surveys per field), frequency (the number of times per year that sampling is conducted) and investigate how the efficacy depends on the dispersal charcteristics of the organism. We will also consider the effect of varying the detection probability. By combining the general spread model with simulations of different survey strategies for specific organisms and landscapes, we aim to improve surveillance strategies and decrease the time to detection after an incursion.

In conclusion, our simulations suggest that an organism's dispersal characteristics influence their spread and ability. Dispersal characteristics should be taken into consideration when designing surveillance strategies. Specifically, the dispersal type and distance impact the rate and patterns of spread and the potential for the invasion to spread to new locations. Additionally, surveillance strategies are critical for early detection. Our results suggest that detecting organisms with non-leptokurtic distributions may be enhanced by increasing survey density (i.e. more surveillance locations), whereas strategies for detecting species with leptokurtic distributions may be improved by increasing the survey frequency (i.e. more surveillance times and area). There is a strong need to incorporate different dispersal characteristics into spread models as species have different dispersal types and distances. Failure to incorporate dispersal type into spread models may underestimate the spread of an invasion with leptokurtic dispersal and result in control or eradication efforts that are ineffective and costly.

\section{ACKNOWLEDGEMENTS}

We thank our funding bodies, Plant Biosecurity Cooperative Research Centre (PBCRC) and the School of Plant Biology at The University of Western Australia. The authors would like to acknowledge the support of the Australian Government's Cooperative Research Centres Programme.

\section{REFERENCES}

Hastings, A., Cuddington, K., Davies, K.F., Dugaw, C.J., Elmendorf, S., Freestone, A., Harrison, S., Holland, M., Lambrinos, J., Malvadkar, U., Melbourne, B.A., Moore, K., Taylor, C., and Thomson, D. (2005) The spatial spread of invasions: new developments in theory and evidence. Ecology Letters, 8(1), 91-101.

Hengeveld, R. (1989) Dynamics of Biological Invasions. Springer, London, UK.

Higgins, S.I., and Richardson, D.M. (1999) Predicting plant migration rates in a changing world: the role of longdistance dispersal. The American Naturalist, 153(5), 464-475.

Kot, M., Lewis, M.A., and van den Driessche, P. (1996) Dispersal data and the spread of invading organisms. Ecology, 77(7), 2027-2042.

Lindström, T., Håkansson, N., and Wennergren, U. (2011) The shape of the spatial kernel and its implications for biological invasions in patchy environments. Proceedings of the Royal Society B: Biological Sciences, 278(1711), 1564-1571.

Mehta, S.V., Haight, R.G., Homans, F.R., Polasky, S., and Venette, R.C. (2007) Optimal detection and control strategies for invasive species management. Ecological Economics, 61 (2-3), 237-245.

Nathan, R., Klein, E., Robledo-Arnuncio, J.J., and Revilla, E., (2012) Dispersal kernels: review, in: Colobert, J., Baguette, M., Benton, T.G., Bullock, J.M. (Eds.), Dispersal Ecology and Evolution. Oxford University Press, Oxford, UK, pp. 186-210.

Peck, S.L. (2004) Simulation as experiment: a philosophical reassessment for biological modeling. Trends in Ecology and Evolution, 19(10), 530-534.

Skellam, J. (1951) Random dispersal in theoretical populations. Biometrika, 196-218.

Simberloff, D., Martin, J.L., Genovesi, P., Maris, V., Wardle, D.A., Aronson, J., Courchamp, F. Galil, B. GarciaBerthou, E., Pascal, M., Pysek, P., Sousa, R. Tabacchi, E., and Vila, M. (2013) Impacts of biological invasions: what's what and the way forward. Trends in Ecology and Evolution, 28(1), 58-66.

Suarez, A.V., Holway, D.A., Case, T.J. (2001) Patterns of spread in biological invasions dominated by longdistance jump dispersal: insights from Argentine ants. Proceedings of the National Academy of Sciences, 98(3), 1095-1100. 